\title{
ChemComm
}

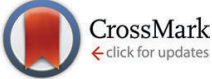

Cite this: Chem. Commun., 2015, 51,5672

Received 6th January 2015 Accepted 19th February 2015

DOI: $10.1039 / \mathrm{c} 5 \mathrm{cc} 00098$

www.rsc.org/chemcomm

\section{Robustness of synthetic circadian clocks to multiple environmental changes $\dagger$}

\author{
Lilia Gurevich, Rivka Cohen-Luria, Nathaniel Wagner and Gonen Ashkenasy*
}

\begin{abstract}
A molecular network that mimics circadian clocks from cyanobacteria is constructed in silico. Simulating its oscillatory behaviour under variable conditions reveals its robustness relative to networks of alternative topologies. The principles for synthetic chemical circadian networks to work properly are consequently highlighted.
\end{abstract}

Complex networks of bio-molecular interactions and chemical transformations allow living cells to rapidly and simultaneously process multiple extracellular input signals. Arguably, among the most central networking features is the regulation of oscillatory behaviour of circadian clocks, endogenous physiological timing systems that generate 24 hour rhythms in anticipation of dusk and dawn. ${ }^{1}$ One of the challenges in contemporary Systems Chemistry research is to mimic such complex functionality using synthetic molecular networks. ${ }^{2}$ However, when looking to fulfil this task, one finds that most currently known biological oscillators are difficult to mimic, due to the complexity of the oscillators themselves and their cellular environments. Yet, it was found nearly a decade ago that the circadian clock of the cyanobacteria Synechococcus elongatus requires neither transcription nor translation, ${ }^{3}$ and is driven by a rather simple core oscillator consisting of just three proteins: KaiA, KaiB, and KaiC. ${ }^{4}$ Mixing these three proteins in vitro with ATP resulted in oscillations with a near-24 hour period in the (total) level of KaiC phosphorylation. In this network, schematically shown in Scheme 1a, KaiC is the only protein with enzymatic activity and functions as autokinase, autophosphatase and ATPase, whereas KaiA and KaiB are regulators of KaiC activity. KaiA stimulates KaiC auto-phosphorylation, while a negative feedback mechanism operates by KaiC inactivation of KaiA in a KaiB-dependent manner (Scheme 1a). ${ }^{4 b, c}$

This commonly observed oscillatory behaviour in biology can be mimicked by a small set of chemical reactions that yield

Department of Chemistry, Ben-Gurion University of the Negev, Beer Sheva 84105, Israel. E-mail: gonenash@bgu.ac.il; Tel:+972-8-6461637

$\dagger$ Electronic supplementary information (ESI) available: (i) Detailed description of simulation, parameters and initial conditions, (ii) additional figures. See DOI: $10.1039 / \mathrm{c} 5 \mathrm{cc} 00098 \mathrm{j}$ oscillations, with the most elaborate systems based on the Belousov-Zhabotinsky (BZ) and similar reactions. ${ }^{5}$ By means of bio-macromolecules, in vitro nucleic acid oscillators have been realized using molecular networking and transcription regulation strategies. ${ }^{6}$ Recently, catalytic replication networks have also served to study emergent phenomena in complex mixtures. ${ }^{2 b, 7}$ Our studies with peptide networks, for example, have shown how these can function in closed or open environments, performing Boolean logic operations, ${ }^{8}$ equivalents to a whole range of chemical logic gates, ${ }^{9}$ and mimicking computational modules and network motifs. ${ }^{8 b, c}$ We now argue that studying de novo designed oscillating reaction networks is very timely and of strong current interest. In a very recent paper, we have utilized a ternary network in an open system, driven by realistic peptide replication processes, to simulate in silico the connectivity and network topology of the KaiABC circadian clock from S. elongatus. This network produced rhythms whose constant frequency was independent of the intake and decay rates, and robust towards concentration fluctuations. ${ }^{8 g}$ Here, we analyse the potential for this network to function as an effective circadian clock under changing physical (temperature, $\mathrm{pH}$ ), chemical (reactant, template and salt concentrations) and experimental (reactant and product fluxes) conditions. Using uniform criteria, we quantitatively characterize the robustness of the clock oscillatory periods and their sustainability over time. The network oscillatory behaviour is then compared with the dynamics of alternate networks, in which catalytic and inhibitory pathways were omitted or diverted (Scheme 1b). Remarkably, we find that by better imitating the Kai system, our native-like clock configuration displays robust behaviour under a wider set of conditions. We suggest that disclosing the dynamic characteristics of circadian clocks in this study will help in the design of synthetic oscillatory devices, as well as of additional devices operating via complex network-dependent functions.

The lead network configuration we exploit to mimic the connectivity in the KaiABC system is shown in Scheme $1 \mathrm{~b}$ (configuration I) and Scheme s1 (ESI $\dagger$ ). This 'native' network effectively functions as an internal clock, and its rhythm is 

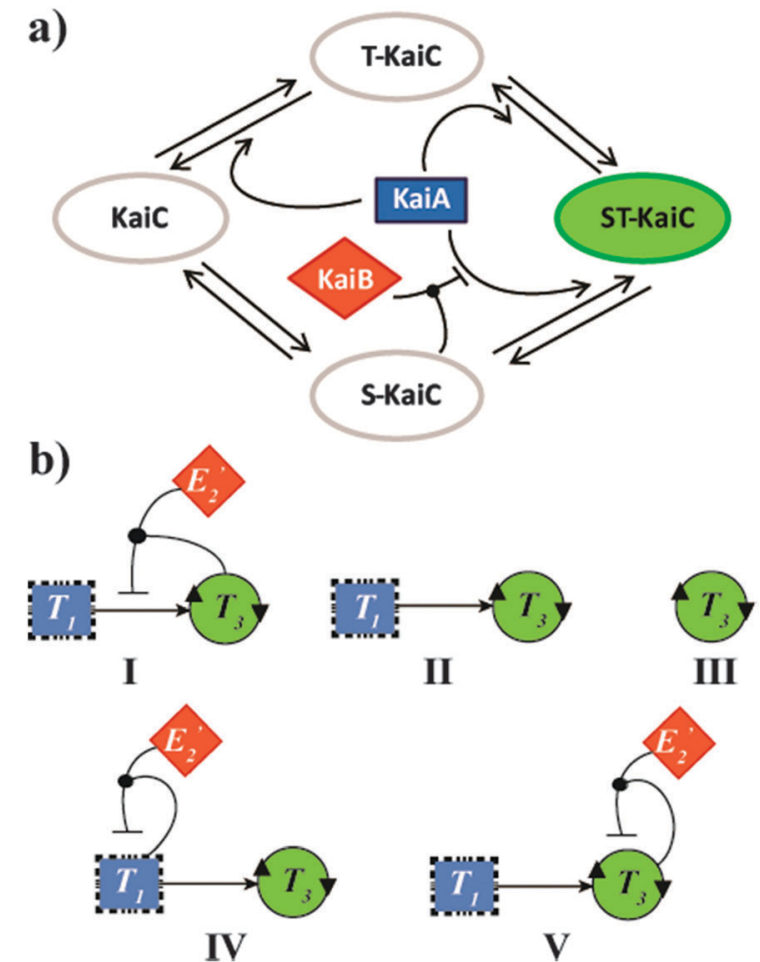

Scheme 1 (a) Schematic description of the KaiABC oscillator of the cyanobacteria S. elongatus. Two residues (S431 and T432) in KaiC undergo autophosphorylation and auto-dephosphorylation. KaiA shifts the equilibrium toward T-KaiC and ST-KaiC. T432 on ST-KaiC spontaneously dephosphorylates and forms S-KaiC. KaiB binds to S-KaiC and inactivates KaiA to shift the equilibrium towards unphosphorylated KaiC. ${ }^{4 b, c}$ (b) Network graph of proposed configurations studied for their potential to reproduce the KaiABC oscillatory behaviour. (I) 'Native' configuration, where the oscillatory production of $T_{3}$, is governed by $T_{3}$ autocatalysis, $T_{3}$ production through cross catalysis by $T_{1}$, and a negative feedback loop due to formation of the $\mathrm{E}_{2}{ }^{\prime} \mathrm{NT}_{3} \mathrm{~T}_{3}$ inhibitory complex. A more detailed diagram showing all reactants, intermediates and products of this network is shown in Scheme s1 (ESI†). (II) Binary configuration consisting of the $T_{3}$ autocatalysis and $T_{3}$ production through cross catalysis by $T_{3}$. (III) A simple configuration consisting only of $T_{3}$ autocatalysis. (IV) A ternary configuration consisting of $T_{3}$ autocatalysis, $T_{3}$ production through cross catalysis by $T_{3}$, and an alternate inhibitory pathway due to formation of the $E_{2}{ }^{\prime} N T_{1} T_{1}$ complex. (V) $A$ ternary configuration consisting of $T_{3}$ autocatalysis, $T_{3}$ production through cross catalysis by $T_{1}$, and an alternate negative feedback loop due to formation of the $\mathrm{E}_{2}{ }^{\prime} \mathrm{NT} \mathrm{T}_{3} \mathrm{~T}_{3}$ complex.

expressed by the oscillatory production of $\mathrm{T}_{3}$ over time (Fig. 1). $\mathrm{T}_{3}$ is formed auto-catalytically and through cross-catalysis by $\mathrm{T}_{1}$, from nucleophilic $(\mathrm{N})$ and electrophilic $\left(\mathrm{E}_{3}\right)$ precursors. The negative feedback of the cross-catalytic production by $\mathrm{T}_{1}$ operates via a competing pathway, where a non-functional electrophile $\left(\mathrm{E}_{2}{ }^{\prime}\right)$ joins a $\mathrm{T}_{1} \mathrm{~T}_{3}$ dimer in forming an inhibiting intermediate. ${ }^{8 g}$

In order to study the kinetics of this circadian network, as well as the kinetics of the alternative networks discussed below (Scheme 1b, configurations II-V), we apply a second order replication model that describes all parallel reactions of three distinct electrophiles $\mathrm{E}_{i}$ and a common nucleophile $\mathrm{N}$, producing three products/templates $\mathrm{T}_{i}$ (eqn (1)-(3); $\left.i, j, k=1,2,3\right)$. The $i=j=k$ cases (eqn (2) and (3)) correspond to autocatalysis, while the $i \neq j$, $k$ cases describe cross catalytic pathways. Importantly, in order to facilitate the oscillatory behaviour, we implement
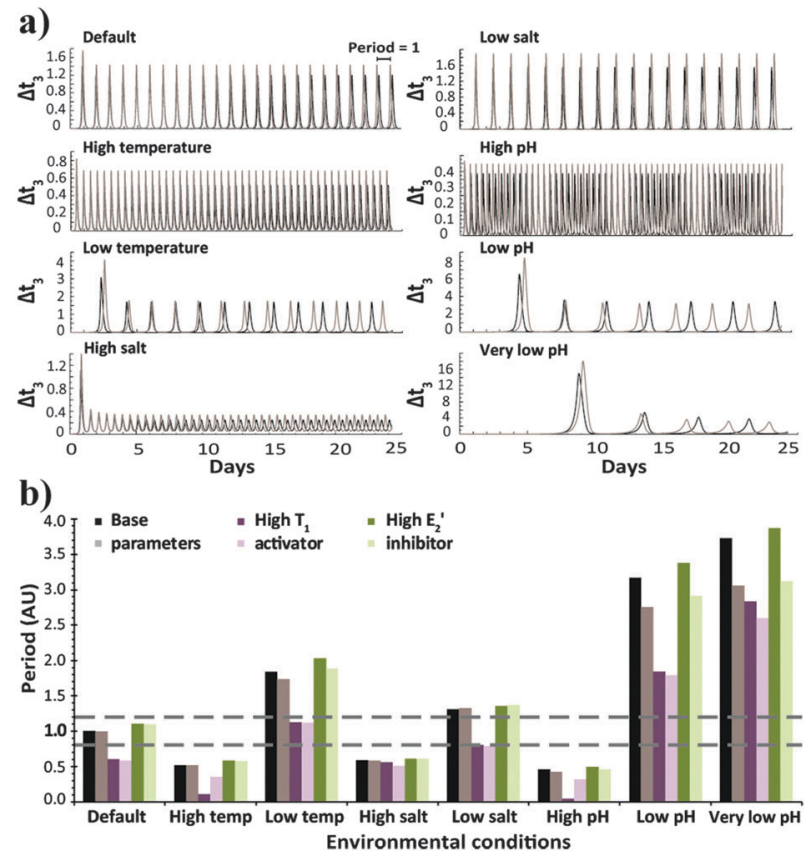

Fig. 1 (a) Time-dependent template concentration for the native configuration, simulated with the base parameters under different environmental conditions. Each panel shows two plots obtained for runs with the basic (black) and 20\% higher (grey) $\mathrm{N}$ and $\mathrm{E}_{3}$ intake and $\mathrm{T}_{3}$ decay rates. (b) Average period values for the native configuration, simulated with the base parameters or when higher amounts of the activator $T_{1}$ or inhibitor $E_{2}^{\prime}$ were introduced. Each set shows bar graphs obtained with the basic (dark colour bars) and 20\% higher (light colour) $\mathrm{N}$ and $\mathrm{E}_{3}$ intake and $\mathrm{T}_{3}$ decay rates. The horizontal dashed lines emphasize conditions resulting in oscillation periods close to 1 . The selection of the relevant parameters that emphasize each of the studied conditions is explained in the ESI $\uparrow$ and specified in Table $s 1$.

the model in open systems, where the reactants $\left(\mathrm{N}\right.$ and $\left.\mathrm{E}_{3}\right)$ have a continuous inflow, and the product $\left(\mathrm{T}_{3}\right)$ decays linearly. ${ }^{10}$ We have investigated this system by simulating the model in Matlab using kinetic parameters relevant for peptide replication experiments. ${ }^{8 b}$ Different network configurations, operating under variable environmental conditions, were assembled by activating, emphasizing and disabling the specific network connections (all together more than 200 different runs; see Table s1 and ESI $\dagger$ for parameter selections).

$$
\begin{gathered}
\mathrm{E}_{i}+\mathrm{N} \stackrel{\mathrm{g}_{i}}{\longrightarrow} \mathrm{T}_{i} \\
\mathrm{E}_{i}+\mathrm{N}+\mathrm{T}_{j} \mathrm{~T}_{k} \underset{\left\langle\mathrm{a}_{j j k}\right.}{\stackrel{\mathrm{a}_{j i k}}{\rightleftarrows}} \mathrm{E}_{i} \mathrm{NT}_{j} \mathrm{~T}_{k} \stackrel{\mathrm{b}_{i}}{\longrightarrow} \mathrm{T}_{i} \mathrm{~T}_{j} \mathrm{~T}_{k} \\
\mathrm{~T}_{i} \mathrm{~T}_{j} \mathrm{~T}_{k} \underset{\left\langle\mathrm{f}_{j j k}\right.}{\stackrel{\mathrm{f}_{j i k}}{\rightleftarrows}} \mathrm{T}_{i}+\mathrm{T}_{j} \mathrm{~T}_{k} \underset{\langle\mathrm{d}\rangle_{j k}}{\stackrel{\mathrm{d}_{j k}}{\rightleftarrows}} \mathrm{T}_{i}+\mathrm{T}_{j}+\mathrm{T}_{k}
\end{gathered}
$$

Fig. 1 shows a series of results obtained for the native circadian network (I) over time, highlighting the oscillatory production of $\mathrm{T}_{3}$ under different temperature, salt concentration and $\mathrm{pH}$ environments. The system under each of these conditions was also analysed when higher amounts of the activator $\mathrm{T}_{1}$ or inhibitor $\mathrm{E}_{2}{ }^{\prime}$ were introduced, or when $\mathrm{E}_{3}$ intake and $\mathrm{T}_{3}$ decay rates (oscillator flux) were increased. While, clearly, $\mathrm{T}_{3}$ displays oscillations under all of the studied conditions, the screening also reveals the effects of each 
condition on the oscillations' period, phase, amplitude and sustainability (Fig. 1a). The oscillation periodicity was calibrated with respect to the sustained period obtained when the network functions under the default conditions (period = 1 day; Fig. 1a). As previously reported for other in vitro circadian clocks, ${ }^{3,4 e, 11}$ we find that substantial changes in conditions can significantly affect the oscillation period. Thus, when the network was studied in environments of high temperatures, high salt concentrations or high $\mathrm{pH}, \mathrm{T}_{3}$ oscillated with shorter periods, and oppositely, at low temperatures, low salt concentrations or low $\mathrm{pH}, \mathrm{T}_{3}$ oscillated with longer periods. As could also be expected, an increase in the activator concentration shortened the periods, and an increase in the inhibitor concentration extended the periods. Remarkably, in some cases, changing two opposing parameters simultaneously resulted in oscillations very similar to that of the default conditions. For example, applying low temperature and high activator concentration resulted in a period of 1.1 (Fig. 1b). This phenomenon also resonated with the temperature dependency and temperature compensation behaviour observed in several studies with the KaiABC ${ }^{11 b, 12}$ and other ${ }^{11,, 13}$ natural oscillating systems.

The robustness of the oscillator period is known to be critical for its function. Still, as apparent from the results of Fig. 1, it is difficult to quantify the robustness over an entire range of environmental conditions using a single parameter. We thus evaluate the period robustness under each specific set of environmental conditions (Fig. 2). The calculated robustness, ranging from 0 to 1 , is defined as the reciprocal of the product of the three ratios, comparing the periods of the oscillations from different fluxes, for the three sets of initial concentrations. For example, simulating the native clock (I) with fluxes that differ by $20 \%$ would be expected to yield significantly different oscillation periods, ${ }^{8 g}$ yet the results (Fig. 1 and 2) show that except for running in high temperature or high $\mathrm{pH}$ environments, this network maintains almost identical $\mathrm{T}_{3}$ periods.

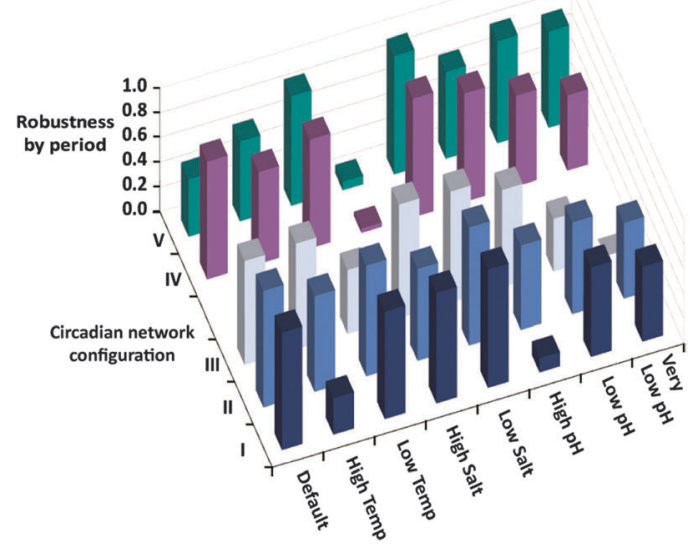

Fig. 2 Robustness by period, calculated for the five different configurations shown in Scheme 1b, under eight different sets of environmental conditions. The robustness is defined as the reciprocal of the product of the three ratios, comparing the periods of the $T_{3}$ oscillations from different fluxes, for the three sets of initial concentrations (base parameters, high $T_{1}$ activator, high $\mathrm{E}_{2}^{\prime}$ inhibitor).
Fig. 2 is then further used to compare the period robustness under all eight sets of environmental conditions, for the five network configurations of Scheme $1 \mathrm{~b}$. This uniform measure indicates that the native configuration (I) is more robust under a wider set of conditions than both the simple autocatalytic reaction (configuration III), and the alternate ternary network (V) where the negative feedback loop is formed by a direct inhibition of the $\mathrm{T}_{3}$ autocatalysis. The binary network (II) consisting of the $\mathrm{T}_{3}$ autocatalysis and $\mathrm{T}_{3}$ production through cross catalysis but no inhibition, and the ternary network (IV) where $\mathrm{T}_{1}$ activity is auto-inhibited, seem to exhibit similar robustness by period to that of the native system.

In order to further compare the performance of the five studied network configurations, we analyse the sustainability of their oscillations over time for variable conditions (Fig. 3). Here again, after simulating the time-dependent behaviour (e.g., Fig. 3a), a uniform criterion is applied, reflecting the ratio between the amplitude of the oscillations at the beginning of the reaction and at the final stages. Fig. 3b shows that under the base parameter set of concentrations, all configurations will produce sustained oscillations. Yet, under high concentration of the activator $\mathrm{T}_{1}$, the native configuration will still oscillate in a sustainable fashion, while the oscillations of configurations II, IV and V will dampen over time. The oscillations of the alternate configuration (V) also dampen when the system functions under high $\mathrm{E}_{2}{ }^{\prime}$ inhibitor concentrations.
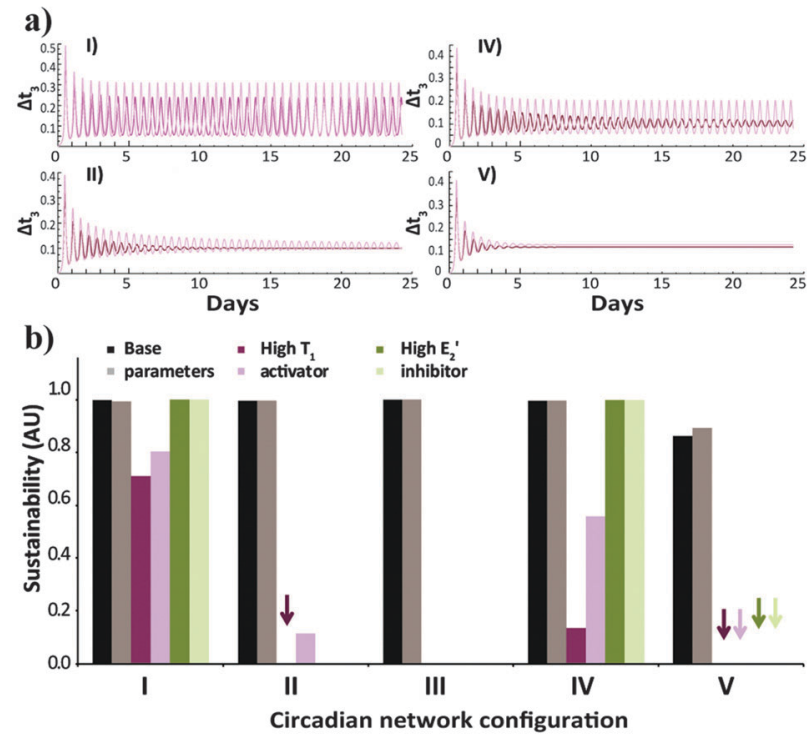

Fig. 3 (a) Time-dependent template concentrations obtained for the various network configurations (I, II, IV, V; Scheme 1b), under the default environmental conditions, for the case of high $T_{1}$ activator concentration. (b) Sustainability values for the different configurations, under default environmental conditions, for the three sets of different concentrations (base parameters, high $\mathrm{T}_{1}$ activator, high $\mathrm{E}_{2}{ }^{\prime}$ inhibitor). The kinetics (a) and bar graphs (b) show results obtained for the basic (dark colour) and 20\% higher (light colour) $\mathrm{N}$ and $\mathrm{E}_{3}$ intake and $\mathrm{T}_{3}$ decay rates. Arrows in (b) mark runs that resulted in oscillatory behaviour with sustainability $\leq 0.05$. Note that no effect of $E_{2}{ }^{\prime}$ increase, or $T_{1}$ and $E_{2}^{\prime}$ increase, is expected for systems II and III, respectively. The selection of the relevant parameters that emphasize each of the studied conditions is explained in the ESI $\dagger$ and specified in Table s1. 
Considering both the period robustness and sustainability results under the various conditions, we suggest a mechanism by which our circadian network works properly. Apparently, the fundamental $\mathrm{T}_{3}$ autocatalytic oscillatory behavior due to intake and decay of $E_{3}$ and $T_{3}$ is not sufficient for robust and sustained oscillations, and thus needs to be stabilized by cross catalysis from $T_{1}$. However, for the activator $T_{1}$ to work properly, a compensation mechanism should be in place in order to keep its concentration low. This is optimally accomplished by the feedback loop, operating via the $\mathrm{E}_{2}{ }^{\prime}$ association with $\mathrm{N}$ and the $\mathrm{T}_{1} \mathrm{~T}_{3}$ dimer - but not with the homo-dimers $\mathrm{T}_{1} \mathrm{~T}_{1}$ or $\mathrm{T}_{3} \mathrm{~T}_{3}-$ which facilitates the siphoning off of excess $\mathrm{T}_{1}$ and thus allows the network to be less sensitive to $\mathrm{T}_{1}$ fluctuations.

In this work we shed light on the design principle and Systems Chemistry of potentially realistic synthetic circadian clocks. While the work is still theoretical in nature, many features of circadian clocks operating under different environmental and experimental conditions were revealed, which would be difficult to predict without the numerical simulation. In that context, it should be noted that no demonstration of a replicating molecular network oscillating out-of-equilibrium has been made available so far. We suggest that this target could be addressed by experimentalists in the near future.

This research is supported by the European Research Council (ERC 259204).

\section{Notes and references}

1 J. Bass, Nature, 2012, 491, 348.

2 (a) R. F. Ludlow and S. Otto, Chem. Soc. Rev., 2008, 37, 101; (b) Z. Dadon, N. Wagner and G. Ashkenasy, Angew. Chem., Int. Ed., 2008, 47, 6128; (c) J. R. Nitschke, Nature, 2009, 462, 736; (d) L. J. Prins, Chim. Ind., 2010, 92, 126; (e) K. Ruiz-Mirazo, C. Briones and A. de la Escosura, Chem. Rev., 2014, 114, 285.

3 M. Nakajima, K. Imai, H. Ito, T. Nishiwaki, Y. Murayama, H. Iwasaki, T. Oyama and T. Kondo, Science, 2005, 308, 414.
4 (a) J. S. Markson and E. K. O'Shea, FEBS Lett., 2009, 583, 3938; (b) G. Dong, Y.-I. Kim and S. S. Golden, Curr. Opin. Genet. Dev., 2010, 20, 619; (c) P. Lenz and L. Sogaard-Andersen, Nat. Rev. Microbiol., 2011, 9, 565; (d) M. Egli and C. H. Johnson, Curr. Opin. Neurobiol., 2013, 23, 1; (e) G. Pattanayak and M. J. Rust, Curr. Opin. Microbiol., 2014, 18, 90.

5 (a) I. R. Epstein and J. A. Pojman, and Editors, An Introduction to Nonlinear Chemical Dynamics: Oscillations, Waves, Patterns, and Chaos, 1998, p. 392; (b) F. Sagues and I. R. Epstein, Dalton Trans., 2003, 1201; (c) A. F. Taylor, Prog. React. Kinet. Mech., 2002, 27, 247; (d) R. Yoshida, Adv. Mater., 2010, 22, 3463.

6 (a) E. Franco, E. Friedrichs, J. Kim, R. Jungmann, R. Murray, E. Winfree and F. C. Simmel, Proc. Natl. Acad. Sci. U. S. A., 2011, 108, E784; (b) K. Montagne, R. Plasson, Y. Sakai, T. Fujii and Y. Rondelez, Mol. Syst. Biol., 2011, 7, 466; (c) M. Weitz, J. Kim, K. Kapsner, E. Winfree, E. Franco and F. C. Simmel, Nat. Chem., 2014, 6, 295.

7 (a) G. Ashkenasy, R. Jagasia, M. Yadav and M. R. Ghadiri, Proc. Natl. Acad. Sci. U. S. A., 2004, 101, 11872; (b) J. J. P. Peyralans and S. Otto, Curr. Opin. Chem. Biol., 2009, 13, 705; (c) A. Vidonne and D. Philp, Eur. J. Org. Chem., 2009, 583; (d) O. Taran and G. von Kiedrowski, Chem. Synth. Biol., 2011, 289; (e) J. Li, P. Nowak and S. Otto, J. Am. Chem. Soc., 2013, 135, 9222.

8 (a) G. Ashkenasy and M. R. Ghadiri, J. Am. Chem. Soc., 2004, 126, 11140; (b) N. Wagner and G. Ashkenasy, Chem. - Eur. J., 2009, 15, 1765; (c) N. Wagner and G. Ashkenasy, J. Chem. Phys., 2009, 130, 164907; (d) Z. Dadon, M. Samiappan, E. Y. Safranchik and G. Ashkenasy, Chem. - Eur. J., 2010, 16, 12096; (e) M. Samiappan, Z. Dadon and G. Ashkenasy, Chem. Commun., 2011, 47, 710; $(f)$ G. Ashkenasy, Z. Dadon, S. Alesebi, N. Wagner and N. Ashkenasy, Isr. J. Chem., 2011, 51, 106; $(g)$ N. Wagner, S. Alasibi, E. Peacock-Lopez and G. Ashkenasy, J. Phys. Chem. Lett., 2015, 6, 60.

9 (a) A. P. de Silva and S. Uchiyama, Nat. Nanotechnol., 2007, 2, 399; (b) E. Katz and V. Privman, Chem. Soc. Rev., 2010, 39, 1835; (c) K. Szacilowski, Chem. Rev., 2008, 108, 3481.

10 (a) K. M. Beutel and E. Peacock-Lopez, J. Chem. Phys., 2006, 125, 024908; (b) K. M. Beutel and E. Peacock-Lopez, J. Chem. Phys., 2007, 126, 125104.

11 (a) R. A. Hut and D. G. M. Beersma, Philos. Trans. R. Soc., B, 2011, 366, 2141; (b) S. Akiyama, Cell. Mol. Life Sci., 2012, 69, 2147; (c) F. Hussain, C. Gupta, A. J. Hirning, W. Ott, K. S. Matthews, K. Josic and M. R. Bennett, Proc. Natl. Acad. Sci. U. S. A., 2014, 111, 972.

12 T. S. Hatakeyama and K. Kaneko, Proc. Natl. Acad. Sci. U. S. A., 2012, 109, 8109.

13 (a) P. Ruoff, M. K. Christensen, J. Wolf and R. Heinrich, Biophys. Chem., 2003, 106, 179; (b) T. Takeuchi, T. Hinohara, G. Kurosawa and K. Uchida, J. Theor. Biol., 2007, 246, 195. 